\title{
CONTROLling THE STROKE OF SHAPE MEMORY ACTUATOR WIRES
}

\author{
Gheorghita, V.; Guempel, P.; Ceron, A. E. \& \\ STRITTMATTER, J.
}

Abstract: In this paper we give a short introduction to elements with shape memory effect and discuss their potential in automotive applications. In the second part of the paper we present a prototype of a new controller for NiTinol wires. The presented mechanism is suitable for applications in various fields, for example automotive industry and medical industry. It is necessary to control the stroke of shape memory alloy actuator in order to set the desired proprieties. The NiTi-Controller is a device designed to control the contraction-elongation of shape memory alloy wires when they are loading a weight. Normally the lengths of the wires used are between 75 and $125 \mathrm{~cm}$, and the maximum contraction-elongation is 5\% of its original length. To protect and control the elongation of the wire we use a microcontroller.

Key words: shape memory alloy, microcontroller, automotive systems, actuators
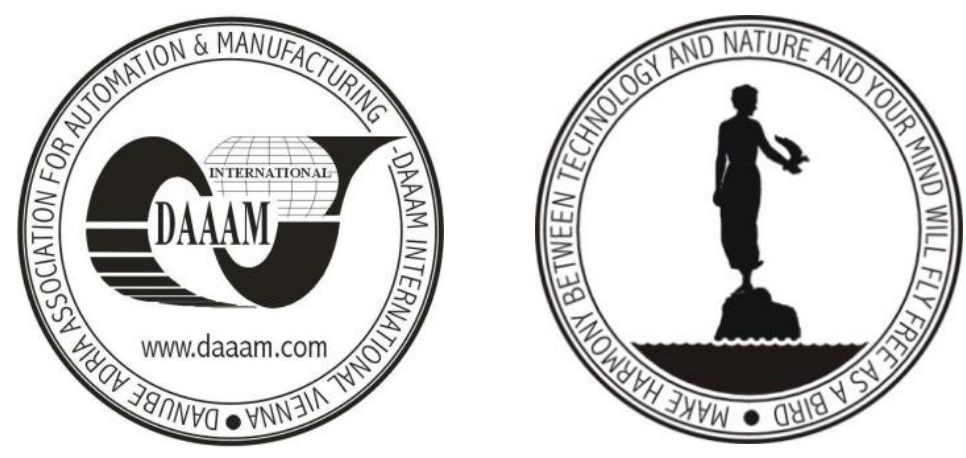

Authors' data: Dipl.-Ing. MSc. Gheorghita, V[iorel]; Prof. Dr.-Ing. Dr.h.c. Guempel, P[aul]*; Dr.-Ing. Srittmatter, J[oachim]; B. Eng. Ceron, A[rturo] E[duardo]; Hochschule Konstanz University of Applied Sciences, Brauneggerstrasse 55, D-78467 Konstanz, Germany; *WITg Institut für Werkstoffsystemtechnik Thurgau an der Hochschule Konstanz, Konstanzer 19, CH-8274, Taegerwilen, Switzerland, vgheorgh@htwg-konstanz.de, guempel@htwg-konstanz.de, arturo.e.ceron@gmail.com, j.strittmatter@witg.ch, joker1@htwg-konstanz.de

This Publication has to be referred as: Gheorghita, V[iorel]; Guempel, P[aul]; Srittmatter, J[oachim] \& Ceron, A[rturo] E[duardo] (2011). Chapter 46 in DAAAM International Scientific Book 2011, pp. 563-572, B. Katalinic (Ed.), Published by DAAAM International, ISBN 978-3-901509-84-1, ISSN 1726-9687, Vienna, Austria DOI: $10.2507 /$ daaam.scibook.2011.46 
Gheorghita, V.; Guempel, P.; Ceron, A.E. \& Strittmatter, J.: Controlling the Stroke...

\section{Introduction}

This application can be used in direction of automotive safety systems and comfort systems. New cars have currently three types of safety systems: passive, precrash and active. In these safety systems shape memory alloys can act as actuators which even can respond in milliseconds (Strittmatter et al., 2009). When these materials are heated the martensitic structure is changed into austenitic structure. During this phase transformation a shape memory actuator is able to contract, to bend or to rotate. The use of shape memory alloys as actuator material has some main advantages: they are smaller and easy to drive with the electrical source and therefore their usage often reduces the complexity of the systems. Thereby weight and size of these systems also can be reduced. Furthermore the shape memory actuators fulfil their function noiseless which is an important advantage concerning applications in the comfort area.

\section{Shape memory alloy actuators}

\subsection{Short introduction about shape memory alloys}

Shape memory alloys (SMA, smart metal, memory metal, memory alloy, muscle wire, smart alloy) are alloys that "remembers" their original, cold-forged shape by returning to the pre-deformed shape by heating. This material is a lightweight, solid-state alternative to conventional actuators such as hydraulic, pneumatic, and motor-based systems. Shape memory alloys are finding applications in industries including medical and aerospace (Gümpel et al., 2004).

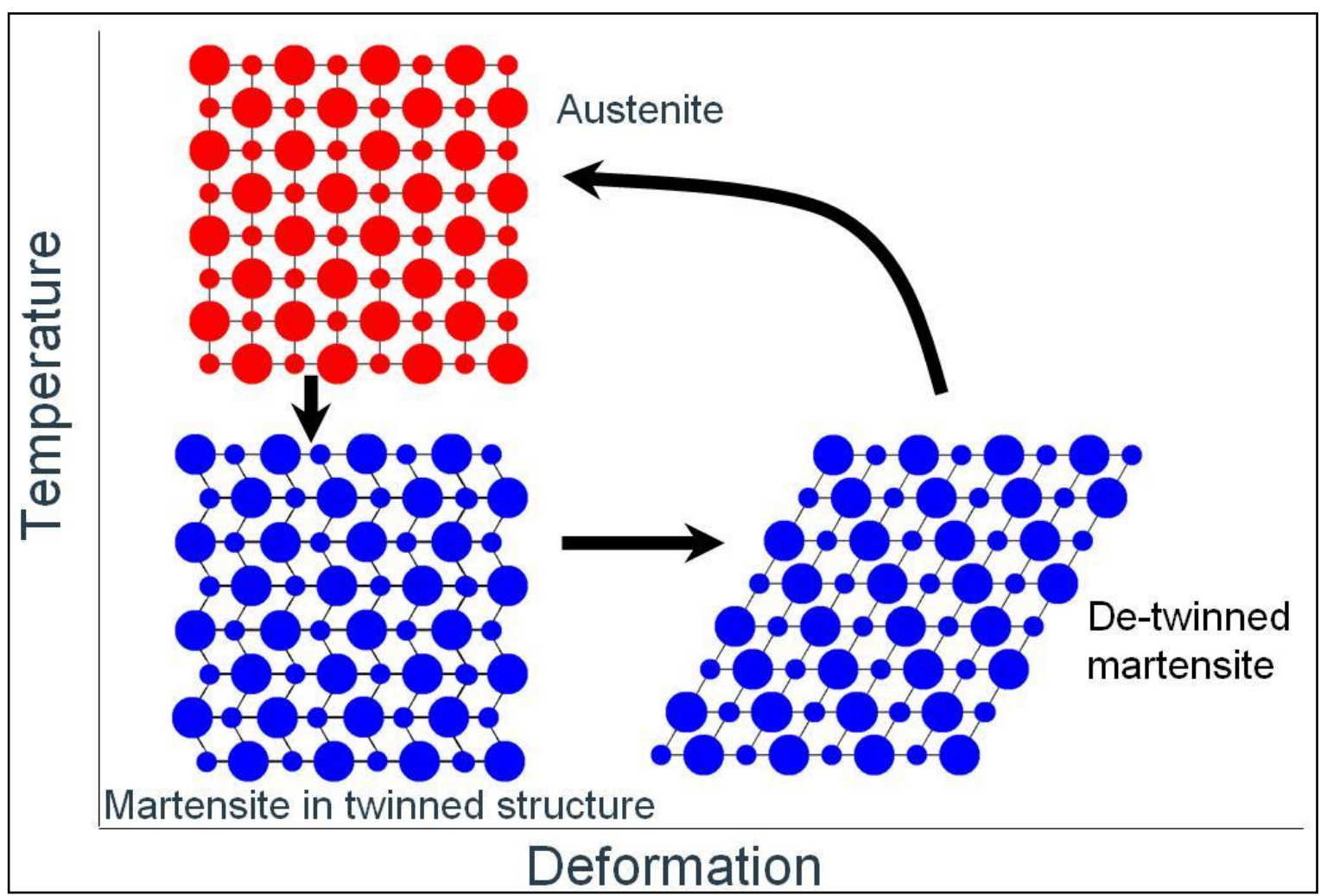

Fig. 1. Martensitic transformation and shape memory effect (Gümpel et al., 2004) 
2.2 Proprieties of the shape memory alloys

Nowadays in engineering the following alloys are used: Nickel-Titanium (NiTi), Copper-Zinc-Aluminium (CuZnAl), Copper-Aluminium-Nickel (CuAlNi) and iron based alloys.

\begin{tabular}{|c|c|c|c|c|c|}
\hline Properties & $\mathrm{NiTi}$ & CuZnAl & CuAINi & FeNiCoTi & $\underset{i}{\mathrm{FeMnS}}$ \\
\hline \multicolumn{6}{|c|}{ Dates regarding shape memory alloy } \\
\hline Minimum PUT $\mathrm{M}_{\mathrm{S}}\left({ }^{\circ} \mathrm{C}\right)$ & -200 & -200 & -200 & -200 & -50 \\
\hline Maximum PUT $A_{F}\left({ }^{\circ} \mathrm{C}\right)$ & $\ldots+120$ & ...+120 & ...+170 & 400 & $\ldots+250$ \\
\hline min. /max. Hysteresis & $2^{(b)} \ldots$ & 5 & 20 & $40 \ldots$ & $40 \ldots$ \\
\hline$A_{F}-M_{S}\left({ }^{\circ} K\right)$ & $80(100)$ & $\ldots 25$ & $\ldots 30$ & 200 & 70 \\
\hline Max. Overheating $\left({ }^{\circ} \mathrm{C}\right)$ & 400 & 200 & 300 & 500 & 450 \\
\hline Max. One way memory effect (\%) & & \multirow{5}{*}{$4 \ldots 5$} & \multirow{5}{*}{$4 \ldots 6$} & \multirow{5}{*}{$1 \ldots 1,5$} & \multirow{5}{*}{2} \\
\hline$N<100$ & $5 \ldots 8$ & & & & \\
\hline$N<100.000$ & $2 \ldots 3,5$ & & & & \\
\hline $\mathrm{N}<1.000 .000$ & $1 \ldots 3$ & & & & \\
\hline $\begin{array}{l}\mathrm{N}<10.000 .000 \\
\text { Max. Cycles }\end{array}$ & $\begin{array}{c}0,5 \\
10^{6} \ldots 10^{7}\end{array}$ & & & & \\
\hline $\begin{array}{l}\text { Max. rev. Elongation }(\%)^{(a)} \\
\text { Max. Cycles }\end{array}$ & \begin{tabular}{|l}
$8 \ldots(10)$ \\
$>10^{7(b)}$
\end{tabular} & $\begin{array}{c}2 \ldots(5) \\
10^{4}\end{array}$ & $\begin{array}{l}1 \ldots 2 \\
10^{3}\end{array}$ & $\begin{array}{l}1,5 \ldots 1,7 \\
10^{2}\end{array}$ & $\begin{array}{l}1,5 \\
10^{1}\end{array}$ \\
\hline \multicolumn{6}{|c|}{ Another Properties } \\
\hline Damping capacity (\%) & 15 & 30 & 10 & 8 & 1 \\
\hline Braking elongation & $30 \ldots 50$ & $10 \ldots 15$ & $5 \ldots 10$ & $2 \ldots 18$ & $5 \ldots 10$ \\
\hline Biocompatibility & Very good & bad & bad & & \\
\hline Corrosion resistance & Very good & weak & good & & \\
\hline
\end{tabular}

Tab. 1. Proprieties of shape memory alloy $(* * *)$

The difference between the heating transition and the cooling transition gives rise to hysteresis where some of the mechanical energy is lost in the process (Gümpel et al. 2004, Kwan \& Nguyen, 2007).

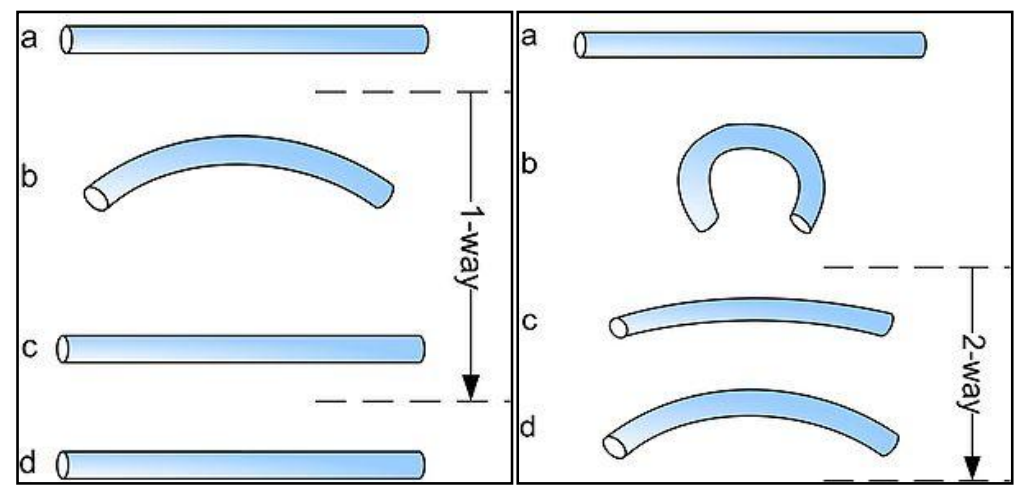

Fig. 2. One and two way effect of shape memory alloy (*)

Shape memory alloys show different shape memory effects: one way, two way and pseudo elasticity. 
The one way shape memory effect takes place when a shape memory alloy is in its cold state (below $A_{s}$ ): the metal can be bent or stretched and will hold those shapes until heated above the transition temperature. Upon heating, the lattice changes to its original lattice structure and therefore to its original shape. When the metal is cooled again it will remain in this "hot" shape, until it is deformed again. Deformation in this case can be used reversibly until $8 \%$.

During the two way shape memory effect the material remembers two different shapes: one at low temperature, and one at the high-temperature. This can also be obtained without the application of an external force (intrinsic two-way effect). The reason that the material behaves so differently in these situations is a special thermomechanical training process (for details see Gümpel et al., 2004).

Pseudo-elasticity propriety occurs in the austenitic temperature. For example, the frames of some eyeglasses are made of shape memory alloy as they can undergo large deformations in their high-temperature state and then instantly revert back to their original shape when the stress is removed. This is the result of pseudo elasticity; the martensitic phase is generated by stressing the metal in the austenitic state and this martensitic phase is capable of large strains. With the removal of the load, the martensitic transforms back into the austenite phase and resumes its original shape (Gümpel et al., 2004).

Shape memory alloy wires have this special propriety to modify the form when the temperature is changed. The activation of the memory effect generally can be realized with thermal or electrical energy. Thermal energy (for example: heat water, heat oil, heat steam) can be a very good solution for heating the wire, when the medium (liquid, gas) is available in the desired quantity and quality. Also the shape memory elements work in a well limited temperature range and will not be overheated. In this sense the wires can be used to reach higher cycle numbers, because the maximum temperature is limited by the heating medium. In contrast, the activation of shape memory elements with electrical energy normally is easier to realize, but has the risk of overheating the elements and causing possible material defects. These defects may have negative influences upon the memory effect and thereby also upon the function of the whole system.

In automobiles a lot of actuators are used that work on electrical, thermal, hydraulic, magnetic or on mechanic principles. Nowadays almost every car producer is developing "electric cars" and first models are already on the market. In this idea for the future much more electrical energy will be available in the car that also can be used for actuator tasks. One main advantage is the fast contraction of shape memory elements, when a huge electrical energy is applied. So called quick-changing actuators can do a good job even in safety systems (Strittmatter et al., 2009). But the high electrical activation implies the risk of overheating the shape memory elements, especially if the actuator should stay in the new position during some time. Today an overheating of these elements is realized by switchers that interrupt the circuit when the desired position is reached. In the case of quick-changing actuators it is even 
necessary to interrupt the electrical circuit before the position is reached because of the high dynamic of the system.

For this purpose a new controller was developed in our group. The idea is first to protect the shape memory alloys from overheating. But the new controller also allows holding the desired end position over a long period of time, controlling the required electrical energy. Additionally this stroke controller facilitates the actuator to move to predefined positions over the contraction distance without damaging the properties of the shape memory elements.

\section{Stroke controller}

\subsection{Introduction}

The NiTi-Controller is a device designed to control the contraction-elongation (stroke) of shape memory alloy wires when a force is applied to the wires (like a loading weight) while it undergoes a temperature change.

The motivation is to do research upon the possibility to use shape memory alloys in automotive safety system. Some conditions for these systems are: to be easy to use (comfort area), to be retractable (pre-crash area), to be faster (crash area).
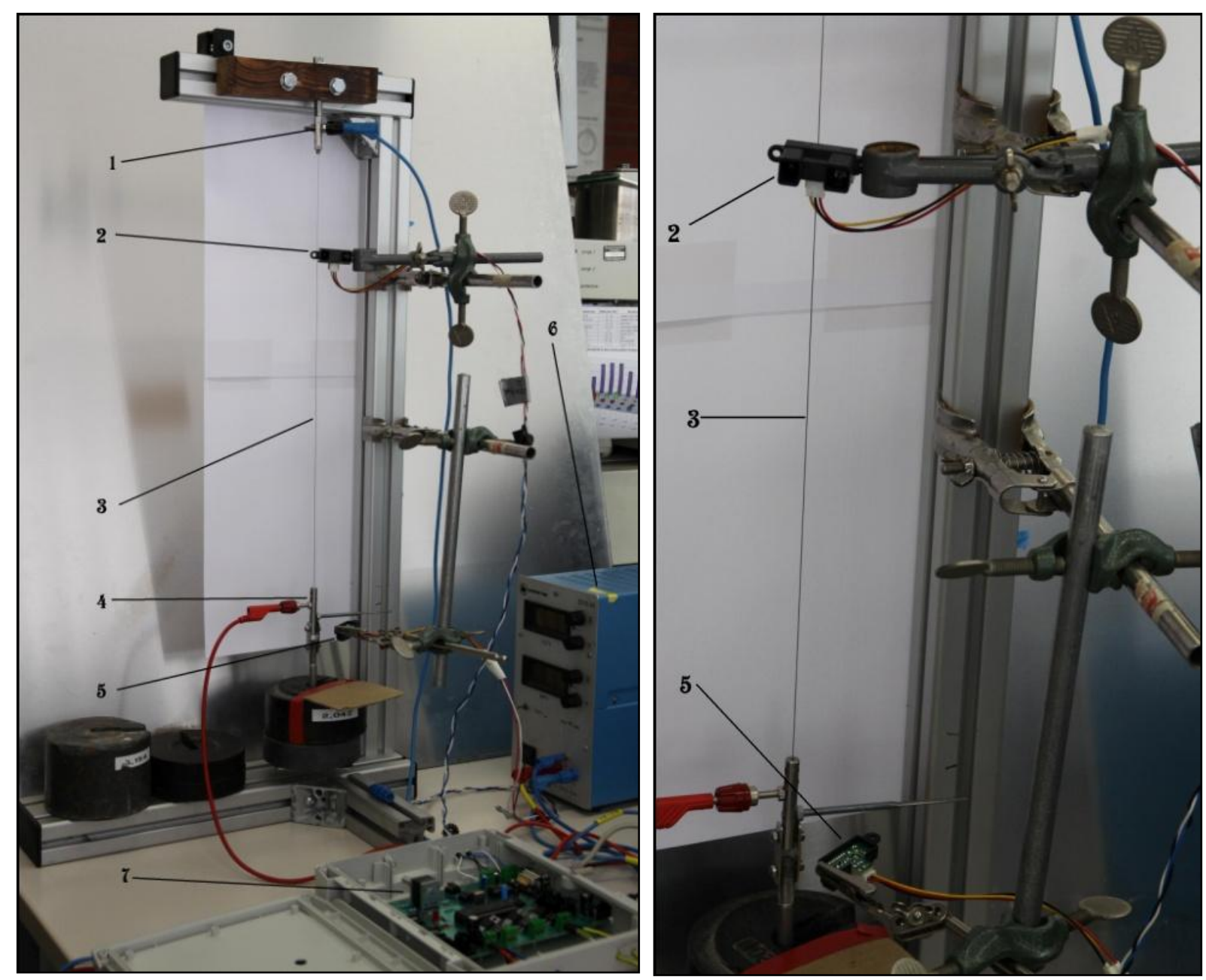

Fig. 3. Demonstrator and sensors 
Gheorghita, V.; Guempel, P.; Ceron, A.E. \& Strittmatter, J.: Controlling the Stroke...

So it is important for the shape memory alloy to control always the displacement. In the next picture is shown a demonstrator, which can control the activating energy for the shape memory alloy wire.

The demonstrator has seven main parts: minus clam (1), distance sensor (2), shape memory alloy wire (3), plus clam (4), displacement sensor (5), energy source (6), controller (7).

This demonstrator has three functions:

- to find the maximum displacement of a wire,

- to set the displacement of the wire and hold it,

- to introduce an external function and change the displacement.

For a given shape memory alloy wire with a certain diameter and length, the maximum displacement can be found with this demonstrator. The wire can contract up to $8 \%$ from the length, depending of the tension. The shape memory wire can be actuated from external information, obtained for example through a distance sensor (2). This application is controlled with a NI LabView program. If we go to the next step with this idea it can be used in automotive safety system (if the power-condition is accomplished). If one of the sensors from the car will "announce" a crash, the information will be conducted to the computer (demonstrator in our case) and the wire (or wires) will contract. This contraction should be very fast, directly proportional with the speed.

The second and third functions are used in the pre-crash phase. When a car runs with a certain speed and is approaching to another vehicle (risk of a possible crash) the computer (in our case demonstrator) will activate the wire. Depending on the computer signal, the wire is able to contract in ranges of $10-100 \%$ of the full stroke. If a crash really happens than the wire (wires) will be contracted to $100 \%$ of the desired stroke.

The line marked with number 1 represents the desired position (could be also a signal from sensor 2), the line marked with number 2 represents the sensor 1 reaction (shape memory alloy stroke reaction).

In fig. 4 are presented sensors and a shape memory alloy wire. The shape memory alloy wire can be actuated from external information, like a distance sensor (2). This application is controlled with a NI LabView program.

So it is possible to introduce a desired position for the wire (the red line form graph) and the shape memory alloy wire will respond with the same value (green line).

This microcontroller is connected to the source and modifies the applying energy for wire, in this case with maximum $24 \mathrm{~V}$ and $3 \mathrm{~A}$. 


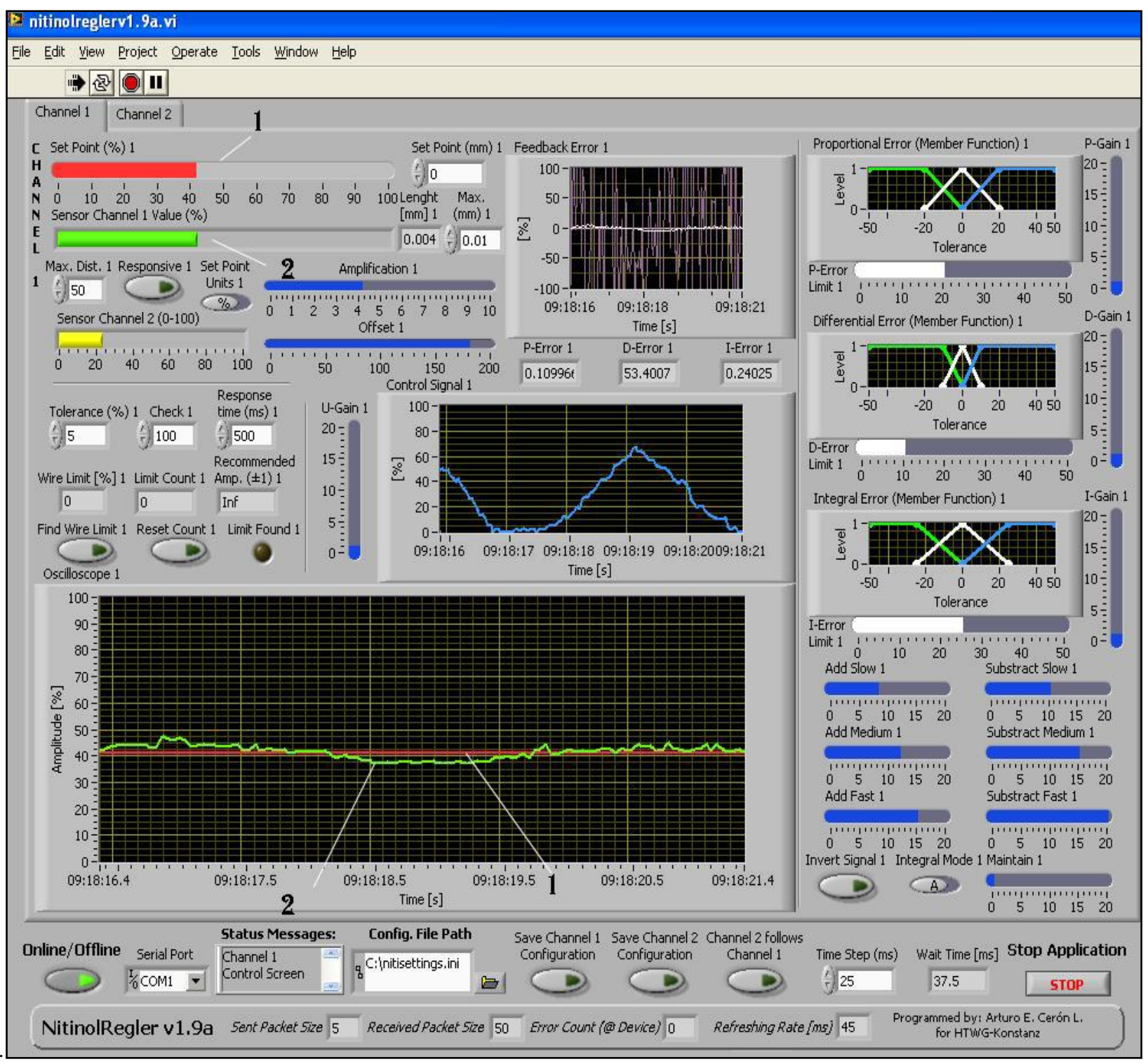

Fig. 4. NI LabView applications - menu display on the monitor screen

\subsection{Hardware}

For the main processing unit, a dsPIC30F4013 microcontroller was used at 30 Mips (millions of instructions per second). A Fuzzy PID (Proportional, Integral and Derivative) controller is implemented in the microprocessor's software, with two integration modes, adjustable time step, adjustable set point, adjustable gains and adjustable triangular member functions. This type of control was used mainly because NiTinol wires present hysteresis in their response, and with the help of independent member functions, this approach is suitable for this kind of application (Kwan \& Nguyen, 2007), also the control becomes friendlier for the user.

As communication protocol, Serial RS-232 is used at 115200 baud to transfer status and configuration data between the microcontroller and the PC. Also it has integrated EEPROM (electrically erasable programmable read-only memory) memory to save configuration settings, which can only be modified by software. At startup, the last configuration used is loaded. 
Gheorghita, V.; Guempel, P.; Ceron, A.E. \& Strittmatter, J.: Controlling the Stroke...

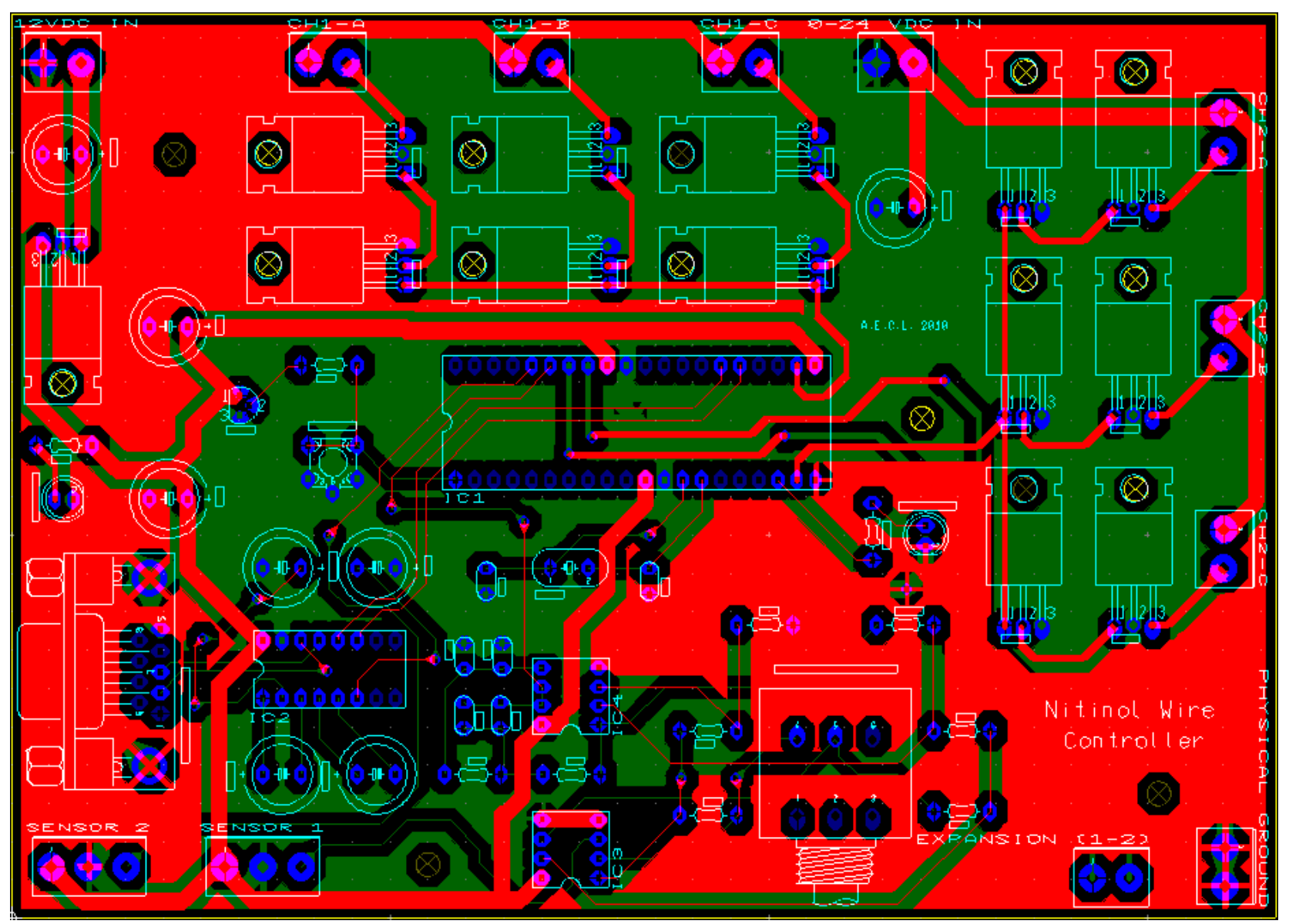

Fig. 5. Circuit card

For current injection, MOSFET (metal oxide semiconductor field-effect transistor) drives were used due to their capability of handling the quantity of current required by the NiTinol wires (Ma \& Song, 2003, Dunlop \& Garcia, 2003). Two channels with three parallel outputs are available.

\subsection{Software}

As for the charts shown in the software, Oscilloscope (for Sensors and Set points), Control Signal, Feedback Error (P, I, and D), and Member Function Setting charts are available for Channel 1 and 2.

Set points can be established manually by the user, or controlled using the channel 2 sensor. According to this information the wire will relax, when an object is far away and contract, when it is coming closer.

Amplification, Offset, PID Gains and Member Function settings can be modified by the user, as well as inverting the signal and selecting between the two integration modes (which can be accumulative or truncated to avoid integral windup).

Another feature was programmed on our software. A new function is available for the user in case he needs help to calibrate the amplification and the offset and to apply current to the wire until the maximum contraction is reached. Upon this value the recommended values of signal amplification for the used wires are calculated.

The user is able to save the configuration settings for channel 1 and 2 ; a configuration file copy is generated in the computer as well. 


\section{Possible application of shape memory alloy actuators in automotive industry}

The present actuators used in modern cars can generally be divided into three categories:

- low power actuators for comfort and bodywork functions

- high power vehicle control actuators

- high frequency engine control actuators

Representing the three categories and plotting the characteristics of the main smart material some of the present actuators can be considered for development of automotive actuators (Auricchio, 2001).

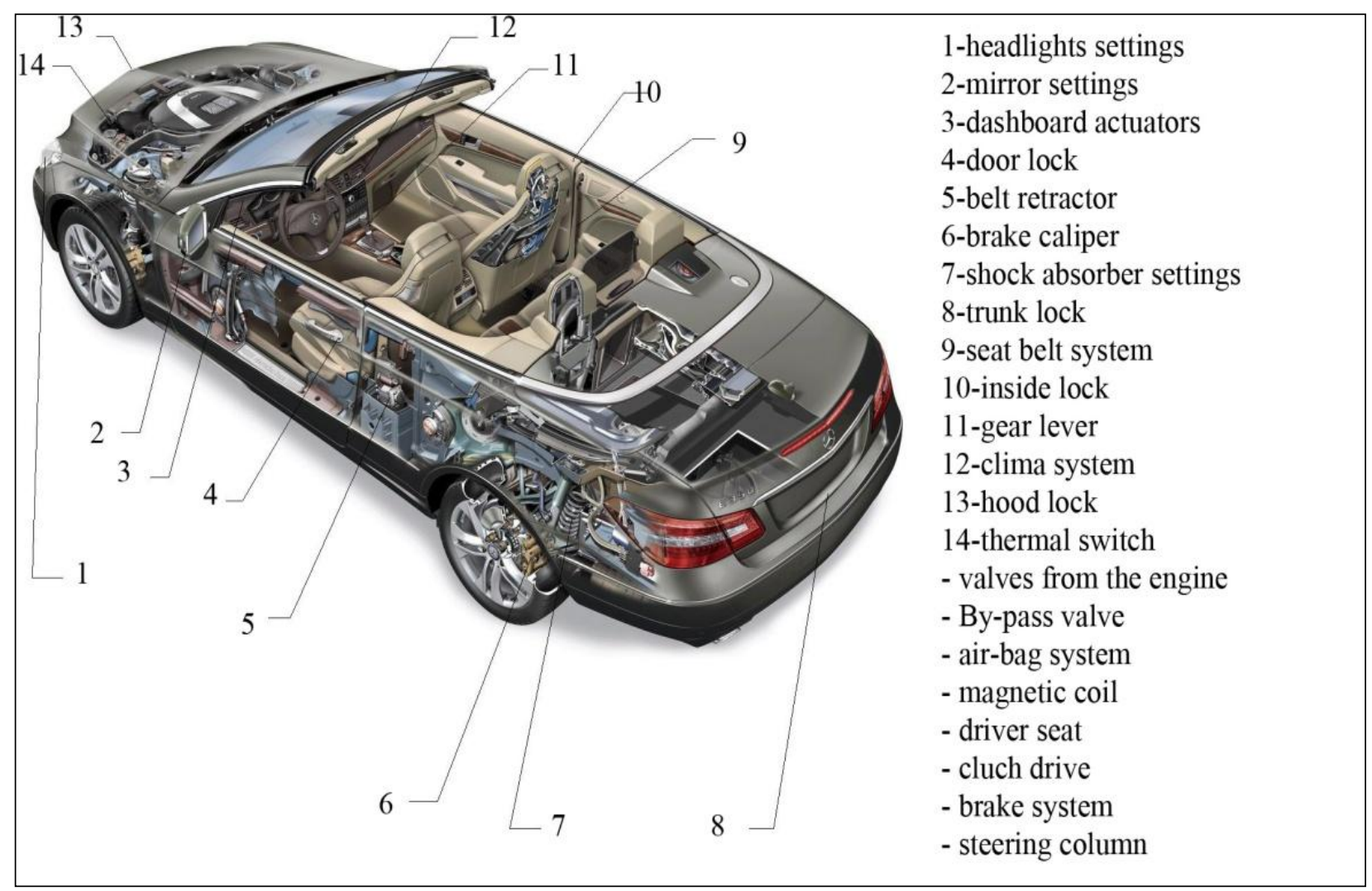

Fig. 6. Possibilities to use SMA in car systems $(* *)$

In automotive industry they can replace electrical, thermal, hydraulic, magnetic actuators from different systems, like safety systems, clutch drive, folding and setting mirror, and others by showing additional advantages.

In safety systems the use of shape memory alloys instead of pyrotechnic or mechanic restrain system has the additional advantage of reversibility. At the same time the quick-changing shape memory elements need a lot of energy for the required short activation time. Therefore it is necessary to use a fast controller. For the future research we need a faster microcontroller which can work at higher voltages.

\section{Conclusions}

A new controller was developed to protect the shape memory alloys from overheating when they are activated in order to hold a desired end position over a 
Gheorghita, V.; Guempel, P.; Ceron, A.E. \& Strittmatter, J.: Controlling the Stroke...

long period of time. The stroke controller fulfils this task applying the minimal electrical energy required for the shape memory elements.

Concerning the present research it can be concluded that

- shape memory alloys have a large range to apply,

- the stroke controller is able to protect the circuit and also the shape memory elements,

- the stroke controller can hold the shape memory wires at maximum elongation without any damage and without any property changes,

- the stroke controller can change the desired position according to the information from an external sensor.

For the use of so-called quick quick-changing shape memory elements in automotive safety systems it is necessary to use faster controllers which can work at higher voltages in order to reach the required activation times of the actuator elements without impairing the properties of the shape memory elements.

\section{References}

Auricchio, F.; Faravelli, L.; Magonette, G. \& Torra, V. (2001), Shape Memory Alloys Advances in Modelling and Applications, International Center for Numerical Methods in Engineering, ISBN 84-89925-82-8, Barcelona

Dunlop, R. \& Garcia, A. (2002). A NiTinol Wire Actuated Stewart Platform, Australasian Conference on Robotics and Automation, pp. 122-123, November 2002, Auckland

Gümpel, P. und 5 Mitautoren (2004), Formgedächtnislegierungen, Expert Verlag, ISBN 3-8169-2293-7, Renningen

Kwan, K. \& Nguyen, B.K. (2007). Position Control of Shape Memory Alloy Actuators Using Self Tuning Fuzzy PID Controller, International Journal of Control, Automation, and Systems, Vol. 4, No. 6, pp. 756-762

Ma, N. \& Song, G. (2003). Control of shape memory alloy actuator using pulse width modulation, Institute of Physics Publishing, Vol. 12, pp. 712-719

Strittmatter, J.; Gümpel, P. \& Zhigang, H. (2009). Long-time stability of shape memory actuators for pedestrian safety system, Journal of Achievements in Materials and Manufacturing Engineering 34/1, pp. 23-30

*** (2011) http://www.thefullwiki.org/Shape_memory_alloy; Accessed on: 2011-0629

*** (2011) http://mercedes-benz-blog.blogspot.com/2010_03_08_archive.html; Accessed on: 2011-06-29

*** (2011) http://memry.com/nitinol-iq/nitinol-fundamentals/physical-properties; Accessed on: 2011-07-25 